Review article

\title{
Clinical performance of glass ionomer cement and composite resin in Class II restorations in primary teeth: A systematic review and meta-analysis
}

\author{
Ana Giselle Aguiar Dias ${ }^{\mathrm{a}, \mathrm{b}}$, Marcela Baraúna Magno ${ }^{\mathrm{c}}$, Alberto Carlos Botazzo Delbem ${ }^{\mathrm{a}}$, \\ Robson Frederico Cunha ${ }^{\mathrm{a}}$, Lucianne Cople Maia ${ }^{\mathrm{c}}$, Juliano Pelim Pessan ${ }^{\mathrm{a}, \mathrm{*}}$ \\ a São Paulo State University (Unesp), School of Dentistry, Araçatuba, Department of Pediatric Dentistry and Public Health, Araçatuba, SP, Brazil \\ ${ }^{\mathrm{b}}$ Department of Dentistry, UNISL - Centro Universitário São Lucas, Porto Velho, RO, Brazil \\ ${ }^{\mathrm{c}}$ Federal University of Rio de Janeiro, Department of Pediatric Dentistry, Rio de Janeiro, RJ, Brazil
}

\section{A R T I C L E I N F O}

\section{Keywords:}

Child

Primary teeth

Glass ionomer cement

Composite resin

\begin{abstract}
A B S T R A C T
Objectives: This study compared the clinical performance of glass ionomer cement (GIC) compared to composite resin (CR) in Class II restorations in primary teeth.

Data: Literature search according to PRISMA guidelines including randomized controlled trials comparing Class II restorations performed with GIC, compared to CR, in primary teeth.

Sources: PubMeb, Scopus, Web of Science, VHL, Cochrane Library, Clinical Trials and OpenGrey, regardless of date or language.

Study: Ten studies were included in qualitative synthesis, and 9 in the meta-analyses (MA). Six studies were classified as low risk of bias, and 4 as "unclear". Heterogeneity ranged from null to high ( $0 \%$ to $73 \%)$. GIC and CR presented similar failure patterns (risk difference $-0.04[-0.11,0.03] ; \mathrm{p}=0.25, \mathrm{I}^{2}=51 \%$ ), and the exclusion of studies with follow-up period $<24$ months, or grouping according to the type of GIC (conventional or resin-modified), or according to the type of isolation (cotton roll or rubber dam), or according to the evaluation criteria applied did not affect the pattern of the results obtained. GIC exhibited significantly lower values of secondary carious lesions (SCL) than CR (SCL: risk difference $0.06[0.02,0.10], \mathrm{p}=0.008, \mathrm{I}^{2}=0 \%$ ). The materials presented similar performance $(\mathrm{p}>0.05)$ regarding the overall effect, as well as for marginal discoloration, marginal adaptation and anatomical form. The superiority of GIC was maintained when resinmodified GIC and rubber dam isolation were analyzed separately.

Conclusions: GIC and CR presented similar clinical performance for all criteria analyzed, except for secondary carious lesions, in which GIC presented superior performance, especially for the resin-modified GIC and with rubber dam isolation.
\end{abstract}

\section{Introduction}

Dental caries is one of the most prevalent diseases in the oral cavity, and its high prevalence is related to inadequate oral hygiene habits and ingestion of carbohydrate-rich foods [1], as well as socioeconomic and behavioral factors. The proximal surfaces are the greatest contributors to the high prevalence of this disease [2], especially in the primary dentition. Since the direct visual inspection of carious lesions in proximal surfaces is impaired by the presence of a contact surface between primary posterior teeth $[3,4]$, more invasive interventions are commonly performed, given that carious lesions in these surfaces are often detected in more advanced stages when compared with smooth surfaces. In these cases, restorative treatment is the most frequently performed.
Although amalgam restorations present high longevity [5], their use has been increasingly discontinued, since they require more invasive operative techniques, demanding wear of intact tooth structure for adequate material retention [6], in addition to concerns related to toxicity and environmental pollution [7]. The aforementioned disadvantages, along with the poor esthetics of amalgam restorations, increased the attention to materials as composite resin (CR) and glass ionomer cement (GIC), due to the greater maintenance of intact tooth structure and their adhesion to the remaining tooth structure. These characteristics allow the use of more conservative restorative techniques, limiting the cavity preparation mainly to decayed tissue removal, thereby preserving the intact tooth structures.

Despite the favorable esthetic and mechanical properties of CRs, the

\footnotetext{
* Corresponding author at: São Paulo State University (Unesp), School of Dentistry, Araçatuba, Department of Pediatric Dentistry and Public Health, Rua José Bonifácio, 1193, 16015050 Araçatuba, SP, Brazil.

E-mail address: jpessan@foa.unesp.br (J.P. Pessan).
} 
Box 1

Search strategy for each database Feb 6th 2018.

\begin{tabular}{|c|c|}
\hline $\begin{array}{l}\text { PubMed } \\
(n=2.154)\end{array}$ & 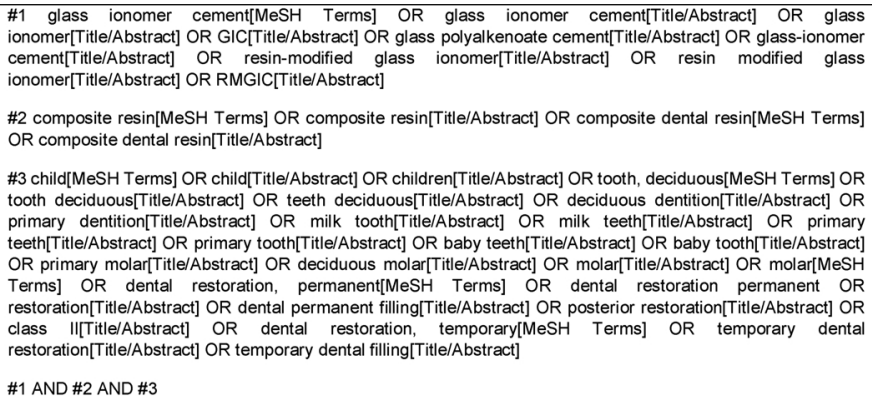 \\
\hline $\begin{array}{l}\text { Scopus } \\
(n=2.138)\end{array}$ & 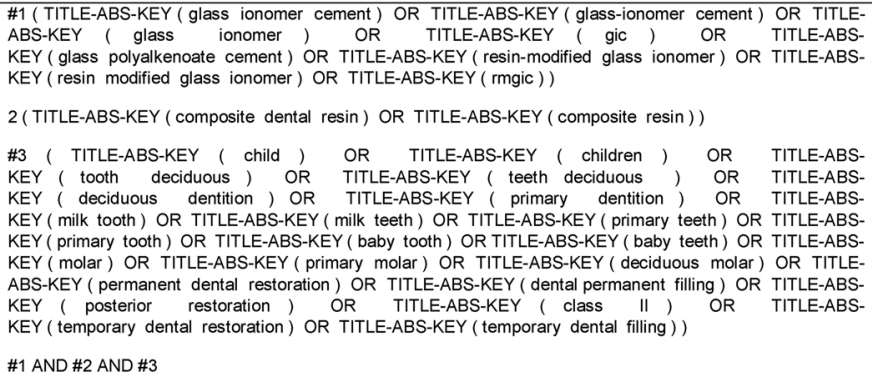 \\
\hline $\begin{array}{l}\text { WEB OF } \\
\text { SCIENCE } \\
(n=725)\end{array}$ & $\begin{array}{l}\text { \#1 Topic:(glass ionomer cement) OR Topic:(glass-ionomer cement) OR Topic:(GIC) OR Topic: (glass } \\
\text { polyalkenoate cement) OR Topic:(glass ionomer) OR Topic: (resin-modified glass ionomer) OR Topic:(resin } \\
\text { modified glass ionomer) OR Topic: (RMGIC) } \\
\text { \#2 Topic:(composite dental resin) OR Topic:(composite resin) } \\
\text { \#3 Topic:(Child) OR Topic: (children) OR Topic: (tooth deciduous) OR Topic: (teeth deciduous) OR } \\
\text { Topic: (deciduous dentition) OR Topic: (primary dentition) OR Topic: (milk tooth) OR Topic: (milk } \\
\text { teeth) OR Topic: (primary teeth) OR Topic: (primary tooth) OR Topic:(baby teeth) OR Topic: (baby tooth) } \\
\text { OR Topic: (primary molar) OR Topic: (molar) OR Topic:(deciduous molar) OR Topic:(permanent dental } \\
\text { restoration) OR Topic: (dental permanent filling) OR Topic: (posterior restoration) OR Topic: (class } \\
\text { II) OR Topic:(temporary dental restoration) OR Topic: (temporary dental filling) } \\
\text { \#1 AND \#2 AND \#3 }\end{array}$ \\
\hline $\begin{array}{l}\text { VHL } \\
(n=206)\end{array}$ & $\begin{array}{l}\text { \#1 (mh:(glass ionomer cement)) OR (tw:(glass ionomer cement)) OR (tw:(glass-ionomer cement)) OR (tw:(glass } \\
\text { ionomer)) OR (tw:(glass polyalkenoate cement)) OR (tw:(GIC)) OR (tw:(resin modified glass ionomer)) OR } \\
\text { (tw:(resin-modified glass ionomer)) OR (tw:(RMGIC)) } \\
\text { \#2 (mh:(composite dental resin)) OR (tw:(composite dental resin)) OR (mh:(composite resin)) OR (tw:(composite } \\
\text { resin)) } \\
\text { \#3 (mh:(child)) OR (tw:(child)) OR (tw:(children)) OR (mh:(molar)) OR (tw:(molar)) OR (tw:(primary molar)) OR } \\
\text { (tw::(deciduous molar)) OR (mh:(tooth, deciduous)) OR (tw:(tooth deciduous)) OR (tw:(teeth deciduous)) OR } \\
\text { (tw:(deciduous dentition)) OR (tw:(primary dentition)) OR (tw:(milk tooth)) OR (tw:(milk teeth)) OR (tw:(primary } \\
\text { teeth)) OR (tw:(primary teeth)) OR (tw:(baby teeth)) OR (tw:((baby tooth)) OR (mh:(dental restoration, permanent)) } \\
\text { OR (tw:(permanent dental restoration)) OR (tw:(dental permanent filling)) OR (tw:(posterior restoration)) OR } \\
\text { (tw:(class II)) OR (mh:(dental restoration, temporary)) OR (tw:(temporary dental restoration)) OR (tw:(temporary } \\
\text { dental filling)) } \\
1 \text { AND \#2 AND \#3 }\end{array}$ \\
\hline $\begin{array}{l}\text { COCHRANE } \\
\text { LIBRARY } \\
(n=431)\end{array}$ & $\begin{array}{l}\# 1 \text { MeSH descriptor: [Glass lonomer Cements] explode all trees; \#2 glass ionomer cement or glass ionomer or } \\
\text { GIC or glass-ionomer cement or glass-ionomer cement; \#3 glass polyalkenoate cement; \#4 resin-modified glass } \\
\text { ionomer or resin modified glass ionomer or RMGIC; } \\
\# 5=\# 1 \text { or \#2 or \#3 or \#4; } \\
\# 9 \text { MeSH descriptor: [Child] explode all trees; \#10 child or children; \#11 \#9 or \#10; \#12 MeSH; descriptor: [Tooth, } \\
\text { Deciduous] explode all trees; \#113 tooth deciduous or teeth deciduous; \#14 \#12 or \#13; \#15 deciduous dentition } \\
\text { or primary dentition; \#16 milk tooth or milk teeth or primary teeth or primary tooth or baby teeth or baby tooth; \#17 } \\
\text { MeSH descriptor: [Molar] explode all trees; \#18 deciduous molar or primary molar or molar; \#19 \#17 or \#18; \#20 } \\
\text { MeSH descriptor: [Dental Restoration, Permanent] explode all trees; \#21 dental restoration permanent or } \\
\text { restoration or dental permanent filling or posterior restoration; \#22 \#20 or \#21; \#23 class II; \#24 MeSH descriptor: } \\
\text { [Dental Restoration, Temporary] explode all trees; \#25 temporary dental restoration or temporary dental filling; } \\
\# 26: \# 24 \text { or \#25; } \\
\# 27=\# 11 \text { or \#14 or \#15 or \#16 or \#19 or \#22 or \#23 or \#26 } \\
\# 6 \mathrm{MeSH} \text { descriptor: [Composite Resins] explode all trees; \#7 composite resin or composite dental resin; } \\
\# 8=\# 6 \text { or \#7 } \\
\# 5 \text { and \#8 and \#27 }\end{array}$ \\
\hline $\begin{array}{l}\text { OPENGREY } \\
(n=0)\end{array}$ & $\begin{array}{l}\text { \#1 glass ionomer cement OR glass-ionomer cement OR GIC OR glass polyalkenoate cement OR glass ionomer } \\
\text { OR resin-modified glass ionomer OR resin modified glass ionomer OR RMGIC } \\
\text { \#2 composite dental resin OR composite resin } \\
\text { \#3 Child OR children OR tooth deciduous OR teeth deciduous OR deciduous dentition OR primary dentition OR } \\
\text { milk tooth OR milk teeth OR primary teeth OR primary tooth OR baby teeth OR baby tooth OR primary molar OR } \\
\text { molar OR deciduous molar OR permanent dental restoration OR dental permanent filling OR posterior restoration } \\
\text { OR class II OR temporary dental restoration" OR temporary dental filling } \\
1 \text { AND \#2 AND \#3 }\end{array}$ \\
\hline
\end{tabular}


restorative technique is more sensitive in relation to the use of GIC, as it involves a greater number of operative steps, combined to the higher sensitivity to moisture of this material. Consequently, in cases in which the use of rubber dam is inviable and/or patient compliance is limited, CR usually is not the first option of restorative material [8], placing GIC as the material of choice. GICs are adhesive materials that release fluoride to the oral environment, and their insertion technique is faster compared to composite resins [9], making this material an important resource for the treatment of children.

Isolated studies present conflicting evidence concerning the longevity of restorations in primary teeth, thus systematic literature reviews, especially involving meta-analysis, are a great tool to aid professionals in clinical decision-making. Within this context, two recent meta-analyses evaluated the performance of restorative materials (adhesive or not) in posterior primary teeth. One study [10] concluded that adhesive materials with resin component (CR, resin-modified GIC and compomer) presented similar longevity rates to each other, with worst performance observed for silver-reinforced GIC. A subsequent study evaluated the effect of GIC restorations in the prevention of marginal carious lesions [11]. The authors concluded that, while the rate of secondary caries was similar among the materials (amalgam, CR, polyacid-modified resin and compomer) for occlusal restorations, the clinical performance of GIC in occluso-proximal restorations was significantly better compared to the other groups.

It is noteworthy, however, that the aforementioned reviews [10,11] gathered data on Class I and II cavities simultaneously, and also included restorative materials that are rarely used or have been increasingly discontinued, which may have largely influenced the results. It is known that the longevity of Class II restorations is significantly reduced compared to Class I preparations [12,13], since the greater number of surfaces involved leads to a greater interface area between the tooth structure and the restorative material, in addition to the loss of the marginal ridge, which poses an occlusal overload on the restoration [14]. Additionally, considering that CR and GIC (conventional or resinmodified, hereafter abbreviated as C-GIC and RM-GIC, respectively) are the materials most widely used in clinical practice compared to other materials available, a direct comparison of the clinical performance of GIC and CR, especially in Class II restorations, might offer relevant information for the treatment of carious lesions in primary posterior teeth. Finally, analysis of the influence of type of GIC (C-CIG or RMGIC) and isolation (rubber dam or cotton roll) on the clinical performance of restorations might also provide relevant information, both for pediatric dentists and public health services.

Thus, this systematic review and meta-analysis evaluated the clinical performance of GIC and CR in Class II restorations in primary molars. As secondary outcomes, the study also evaluated the influence of type of GIC and isolation (rubber dam or cotton roll) on the clinical performance of restorations.

\section{Material and methods}

\subsection{Protocol and registry}

This study was registered in database PROSPERO (registry CRD42015027751) and followed the PRISMA guidelines on the Preferred Reporting Items for Systematic reviews and Meta-Analyses [15].

\subsection{Search strategy}

An electronic search was performed on the following databases: PubMeb, Scopus, Web of Science, Virtual Health Library (VHL), OpenGrey, Clinical Trials and Cochrane Library. A specialized librarian guided the entire electronic search strategy. Hand search was also performed to identify manuscripts that might not have been retrieved by the electronic search.
To find unpublished or ongoing studies, the registry of clinical trials was investigated on the website ClinicalTrials.gov (www.clinicaltrials. gov), without restriction as to date or language of publication. Additionally, the grey literature (produced at governmental, academic, entrepreneurial and industrial levels, in printed or electronic format, yet not controlled by commercial publishers) was searched using the grey literature database OpenGrey (http://www.opengrey.eu/http:// www.opengrey.eu/).

The search strategy, as well as the date of search for all databases, is presented in Box 1. This search strategy was properly adapted to each database.

\subsection{Eligibility criteria}

The study included randomized controlled clinical trials comparing the clinical performance of Class II restorations performed with composite resin (CR) and conventional or resin-modified glass ionomer cement (C-GIC or RM-GIC, respectively) in primary teeth, in children of any age, according to the PICOS strategy described below:

(P) - Population: children with need of Class II restorations in primary teeth;

(I) - Intervention: use of conventional (chemically cured) or resinmodified (light cured) glass ionomer cement;

(C) - Comparison: use of composite resin;

(O) - Outcome: the primary outcome refers to the clinical performance of restorations, evaluated according to the presence of secondary carious lesions, marginal discoloration, marginal adaptation, longevity, retention and wear of restorative material, and anatomical form. The secondary outcomes included the influence of the type of GIC (C-GIC or RM-GIC) and isolation (rubber dam or cotton roll) on the primary outcome;

(S) - Study design: randomized controlled clinical trials.

Editorial letters, pilot studies, historical reviews, in vitro, cohort, observational and descriptive studies, case reports and case series were excluded. The study also excluded investigations evaluating other types of cavities (Class I, III, IV and V) and analyses on polyacid-modified composite resins (compomers) and silver-reinforced GIC as restorative materials.

\subsection{Study selection and data extraction process}

Papers appearing in more than one database were considered only once. Two reviewers (AGAD and MBM) independently analyzed the titles and abstracts of papers found on the databases. Potentially eligible papers were read in full text to clearly determine their eligibility. Data were extracted using a form based on other systematic reviews, in which the following data were recorded: study details (year of publication and authors); criteria for definition and evaluation; details of study methods (study design and follow-up period); details of participants (mean age and number of patients); details of restorative materials employed (restorative protocols: isolation methods and restorative material), and results.

\subsection{Risk of bias in individual studies}

The methodological quality and risk of bias of the included studies were analyzed by two independent reviewers (AGAD and MBM), using the Cochrane Collaboration tool for analysis of risk of bias (http:// handbook.cochrane.org). The evaluation criteria comprised six items: random sequence generation, allocation concealment, blinded evaluations of results, blinding of participants and staff, results with incomplete data, selective report of outcome, and other possible sources of bias. The six domains were evaluated and the included studies were classified. During evaluation of the risk of bias, any divergences between reviewers were solved by discussion and consensus and, if necessary, with the aid of a third reviewer (LCM). 
For each aspect of quality analysis, the risk of bias for each domain was identified following the recommendations of the Cochrane Handbook for Systematic Reviews of Interventions 5.1.0 (http:// handbook.cochrane.org). Each criterion was scored as "yes", indicating low risk of bias; "no", indicating high risk of bias; and "unclear", indicating lack of information or uncertainty about the potential of bias.

Only four among the six Cochrane domains were considered as key domains to evaluate the risk of bias. The studies were considered as "low" risk of bias if there was adequate randomization, allocation concealment, incomplete data and selective reporting. The two domains evaluating blinding (operators, participants and examiners) were not considered as key domain due to differences both in the operative technique and in the clinical aspect of materials. When the study was scored as "unclear" in the key domains, attempts to contact the authors (one weekly contact, for up to 4 weeks) were made to achieve additional information and allow definitive scoring as "yes" or "no".

\subsection{Meta-analyses}

Data on included studies were obtained and analyzed using the software Revman 5.3 (Review Manager v. 5, The Cochrane Collaboration; Copenhagen, Denmark). Eleven meta-analyses (MAs) were performed to evaluate:

(1) The percentage of failure of restorations in all selected studies;

(2) The percentage of failure of restorations in studies with follow-up period equal to or greater than 24 months;

(3) The percentage of failure of restorations, subgrouping by type of GIC: Subgroup 1: C-GIC x CR; Subgroup 2: RM-GIC x CR;

(4) The percentage of failure of restorations, subgrouping by type of isolation: Subgroup 1: rubber dam isolation; Subgroup 2: cotton roll isolation;

(5) The clinical performance of the main parameters (marginal adaptation (MA), marginal discoloration (MD), anatomical form (AF) and secondary carious lesions (SCL)) considering all selected studies;

(6) The clinical performance of the main parameters (MA, MD, AF and SCL) considering studies with follow-up period equal to or greater than 24 months;

(7) The clinical performance of the main parameters (MA, MD, AF and SCL) including only studies using RM-GIC;

(8) The clinical performance of the main parameters (MA, MD, AF and SCL) including only studies using C-GIC;

(9) The clinical performance of the main parameters (MA, MD, AF and SCL) including only studies using rubber dam isolation;

(10) The clinical performance of the main parameters (MA, MD, AF and SCL) including only studies using cotton roll isolation.

(11) The percentage of failure of restorations, subgrouping by type of evaluation criteria applied: Subgroup 1: USPHS criteria; Subgroup 2: FDI criteria, subgroup 3: Serpa et al., 2017 criteria.

According to the criteria for the evaluation of restorations, data on the main parameters analyzed were dichotomized as "acceptable" (restorations without need of replacement or repair) or "unacceptable" (restorations presenting failures or requiring repair or replacement), as shown in Table 1. The prevalence of unacceptable (events) and the total number of restorations per group were used to calculate the risk difference, at a confidence interval of $95 \%$. The random effect was applied and the heterogeneity (intrinsic divergence among studies) was evaluated by the $\mathrm{I}^{2}$ index. Sensitivity analysis was further conducted to estimate and verify the influence of studies, one by one, on the pooled results when the heterogeneity was moderate or considerable (30\%-100\%) [33].

\section{Results}

\subsection{Selection of studies}

After reading the titles and abstracts, 2722 duplicates were removed, with identification of 2937 studies. Among the 25 potentially eligible studies (read in full text), 15 were excluded due to the following aspects: lack of adequate control (studies without control group) $(n=2)$, retrospective study $(n=1)$, sample overlapping $(n=1)$, microorganisms count as the main response variable $(n=1)$, study protocols without results $(n=2)$, presentation of the results of class I and class II restorations without distinction between the two types of cavities $(n=2)$, presentation of the results of permanent and primary teeth without distinction between the two dentitions $(n=1)$ and restorations in permanent teeth $(n=5)$. Thus, ten papers remained for the qualitative synthesis, and nine for the quantitative analysis. The flowchart of study selection is described in Fig. 1 .

\subsection{Characteristics of studies included}

The characteristics of the 10 studies are listed in Table 2. The follow-up period of Class II restorations in primary teeth in the included studies ranged from 6 to 48 months. The design of all 10 papers analyzed comprised randomized clinical trials. Six studies presented splitmouth design $[12,16,18,19,22,24]$ and five studies had parallel design $[17,20,21,23]$. Seven studies applied USPHS criteria [12,16,17,19-22], two applied the FDI criteria $[18,23]$ and one study applied its own criteria [24]. The number of restorations ranged from 75 to 344 , and the number of participants in each study varied from 31 to 180 children, aged 3 to 11 years. Seven papers reported restorations performed using rubber dam isolation [12,17,18,20-23], while 3 studies reported the use of cotton roll isolation [16,19,24].

\subsection{Risk of bias within studies}

For qualitative analysis, the study assessed six domains described in the evaluation of risk of bias of randomized clinical trials, using the Cochrane collaboration tool (http://handbook.cochrane.org). Randomization, secrecy of allocation, incomplete data and selective report were considered as the key domains. After contact with the authors whose papers received unclear scoring in one or more criteria, six studies [12,17,18,22-24] were considered as having low risk of bias in the final score, while 4 papers were scored as unclear [16,19-21].

Concerning blinding during the evaluation of restorations, 3 studies [16,17,22] did not perform blinding, other 3 studies [19-21] did not describe the observation of this aspect, and 4 studies [12,18,23,24] presented low risk of bias. Nine studies presented low risk of bias in the incomplete data criterion, as well as "selective report of outcome" (Table 3). The study of Fuks et al. [20] exhibited sample loss greater than $50 \%$, and thus was scored as high risk of bias.

The risk of bias of the 10 studies selected is presented in Table 3 .

\subsection{Synthesis of results: meta-analysis}

In all MAs, only studies with available data for each parameter analyzed were included, so that MAs with different number of studies are presented. For studies reporting the use of more than one composite resin (CR), both resins were considered. The total number of CR failures (events) are represented by the sum of the number of CR restorations that failed in both groups (number of failures in CR $1+$ number of failures in CR2 = events), and the total number of restorations per group were represented by the sum of the number of teeth in both groups (number of teeth in CR1 + number of teeth in CR2 = Total). No study used more than one type of GIC. The random effect was applied, and a confidence interval of 95\% (IC 95\%) was adopted for all analyses.

In MAs evaluating the percentage of failures (Fig. 2 and Table $4-$ 
Table 1

Dichotomy of results according to the evaluation criteria of the studies.

\begin{tabular}{|c|c|c|c|c|c|c|}
\hline \multirow[t]{2}{*}{ Parameters } & \multicolumn{2}{|c|}{ USPHS modified criteria $[12,16,17,19-22]$} & \multicolumn{2}{|c|}{ FDI World Federation criteria $[18,23]$} & \multicolumn{2}{|c|}{ Serpa et al. criteria [24] } \\
\hline & Acceptable & Unacceptable & Acceptable & Unacceptable & Acceptable & Unacceptable \\
\hline \multirow[t]{3}{*}{ Failure } & & & $1,2,3$ & 4,5 & & $1,2,3,4$ \\
\hline & & & & & \multicolumn{2}{|c|}{ radiographic criteria } \\
\hline & & & & & $\begin{array}{l}0,1 \\
\text { clinical crit }\end{array}$ & $2,3,4$ \\
\hline Marginal adaptation & Alpha Bravo & Charlie Delta & $1,2,3$ & 4,5 & & \\
\hline Marginal discoloration & Alpha Bravo & Charlie Delta & $1,2,3$ & 4,5 & & \\
\hline Anatomical Form & Alpha Bravo & Charlie & $1,2,3$ & 4,5 & & \\
\hline Secondary caries & Alpha & Bravo Charlie Delta & $1,2,3$ & 4,5 & & \\
\hline
\end{tabular}

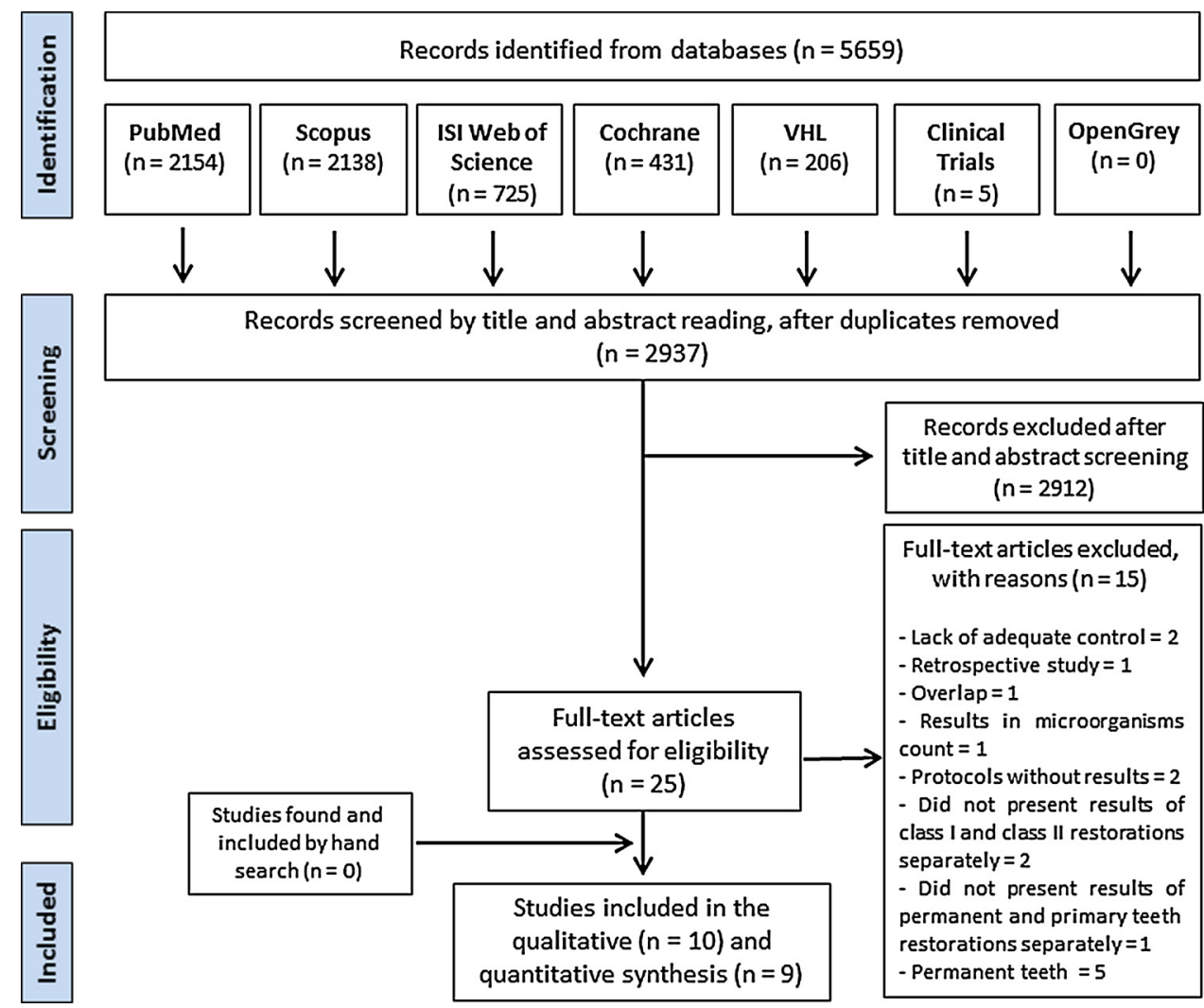

Fig. 1. Flowchart of study selection, presenting the number of studies identified, eligible and included in the review.

Analyses 1, 3, 5, 8 and 11), the heterogeneity ranged from inexistent to moderate $(51,73,51,51$ and $51 \%$, respectively) (http://handbook. cochrane.org/). To reduce heterogeneity, the selective removal of studies one by one was performed, and the heterogeneity ranged from 0 to $57 \%$ in analyses $1,5,8$, and 0 to $81 \%$ in analysis 3 . However, given that the removal of studies did not influence the results, no study was removed from the final MAs.

In six MAs, there was no significant difference between the two materials (CR or GIC). Also, the exclusion of studies with follow-up shorter than 24 months (Table 4 - Analysis 3) did not alter the final results (RD $\left.-0,06[-0.20,0.07] ; p=0.36 ; I^{2}=73 \%\right)$. Six studies [12,16-18,20,23] used RM-GIC, while three studies [19,21,24] reported the use of a C-GIC. Individual analysis per type of GIC compared to $\mathrm{CR}$ did not demonstrate the superiority of RM-GIC (RD -0.02 $[-0.08,0.03] ; \mathrm{p}=0.36 ; \mathrm{I}^{2}=0 \%$ ), or of C-GIC (RD $-0.12[-0.38$, $0.15] ; \mathrm{p}=0.39 ; \mathrm{I}^{2}=86 \%$ ), in relation to failures compared to $\mathrm{CR}$ (Table 4 - Analysis 5).

In MAs assessing the clinical performance of restorations (Figs. 3-5, Table 4 - Analyses 2, 4, 6, 7, 9, 10), the heterogeneity ranged from null to moderate $(27,45,0,0,20$ and $34 \%$, respectively). After removing studies one by one, the heterogeneity ranged from 0 to $57 \%$ in analysis 4 , and from 0 to $49 \%$ in analysis 10 . Given that the removal of studies did not influence the results (to adopt null heterogeneity $\left(\mathrm{I}^{2}=0 \%\right)$ ), no study was removed from the final MAs.

Four out of the 6 MAs evaluating the clinical performance (Figs. 3-5; Table 4 - Analyses 2, 4, 6 and 10) presented the same standard of final outcome: GIC exhibited significantly better clinical performance than $\mathrm{CR}$ in relation to the prevalence of secondary carious lesions, and similar performance concerning the other parameters. The exclusion of studies with follow-up shorter than 24 months (Table 4 Analysis 4) did not alter the trend observed for the MA assessing all studies that were initially selected.

Regarding the influence of the type of GIC on the clinical performance of restorations, the same pattern described above was observed on the MA considering only studies using RM-GIC (Fig. 4). However, when only studies using C-GIC were grouped (Fig. 4), the clinical performance of this material was similar to that of CR for all parameters analyzed, including the occurrence of secondary carious lesions. The difference in clinical performance (IC 95\%) in the final analysis ("overall effect") between RM-GIC and CR, and between C-GIC and CR, 


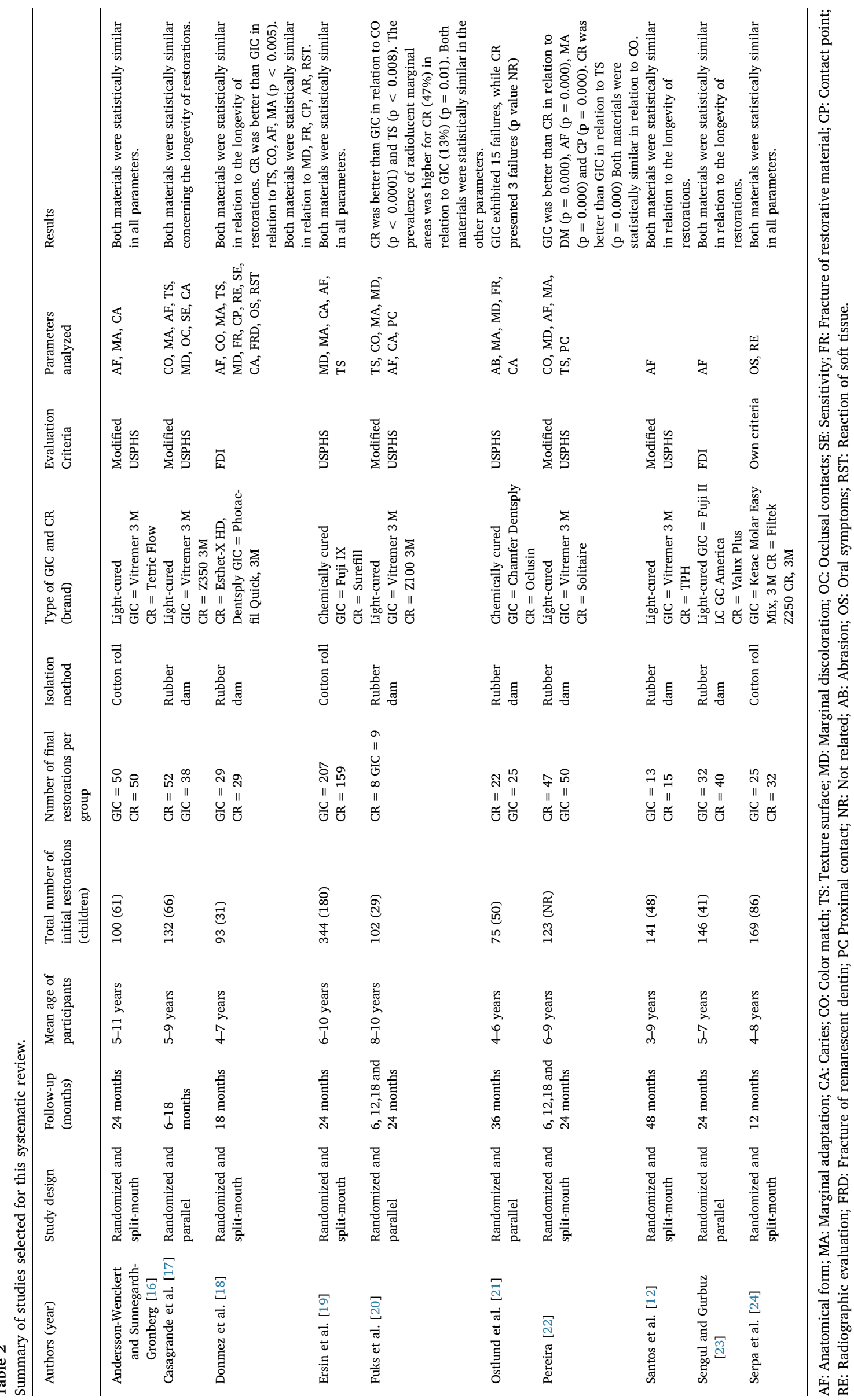


Table 3

Risk of bias of included studies.

\begin{tabular}{|c|c|c|c|c|c|c|}
\hline $\begin{array}{l}\text { Author, year of } \\
\text { publication }\end{array}$ & $\begin{array}{l}\text { Random sequence } \\
\text { generation* }\end{array}$ & $\begin{array}{l}\text { Allocation } \\
\text { Concealment* }\end{array}$ & $\begin{array}{l}\text { Blinded evaluations of } \\
\text { results }\end{array}$ & $\begin{array}{l}\text { Blinding of participants } \\
\text { and staff }\end{array}$ & $\begin{array}{l}\text { Results with } \\
\text { incomplete data * }\end{array}$ & $\begin{array}{l}\text { Selective report of } \\
\text { outcome* }\end{array}$ \\
\hline $\begin{array}{l}\text { Andersson-Wenckert } \\
\text { et al. [16] }\end{array}$ & - & $?$ & - & - & + & + \\
\hline Casagrande et al. [17] & + & + & - & - & + & + \\
\hline Donmez et al. [18] & + & + & + & - & + & + \\
\hline Ersin et al. [19] & $?$ & $?$ & $?$ & - & + & + \\
\hline Fuks et al. [20] & + & ? & ? & - & - & + \\
\hline Ostlund et al. [21] & ? & ? & ? & - & + & + \\
\hline Pereira [22] & + & + & - & - & + & + \\
\hline Santos et al. [12] & + & + & + & - & + & + \\
\hline Sengul and Gurbuz [23] & + & + & + & - & + & + \\
\hline Serpa et al. [24] & + & + & + & - & + & + \\
\hline
\end{tabular}

Risk of bias: low $(+)$, high ( - ), or unclear (?).

Key domains (*).

was RD $0.03[0.00,0.06](\mathrm{p}=0.06)$ and $\mathrm{RD}-0.02[-0.07,0.04]$ $(\mathrm{p}=0.56)$, respectively.

Finally, concerning the influence of type of isolation, the performance of GIC on SCL, as well as on the final analysis ("overall effect"), was significantly better than CR when procedures were performed using rubber dam isolation, without significant differences for the other parameters (Fig. 5). For procedures performed with cotton roll isolation (Fig. 5), no significant differences were observed between materials for any parameter analyzed.

Numerical data on the 11 meta-analyses are described in Table 4.

\section{Discussion}

The conflicting evidence of isolated clinical studies regarding the longevity of restorations in primary teeth, especially in occluso-proximal cavities, complicates the selection of restorative materials for clinicians, which encouraged the present systematic review. In general, the present results demonstrated that the clinical performance of the two restorative materials assessed (GIC and CR) was similar for most clinical parameters analyzed (marginal discoloration, marginal adaptation, retention of restoration and wear of the restorative material) in Class II restorations in primary teeth. However, regarding the occurrence of secondary carious lesions, GIC restorations presented significantly better clinical performance than that observed for CR restorations. To allow a deeper interpretation of data obtained from the primary studies included in this review, 11 meta-analyses were performed with different objectives, which are discussed in detail below.

The similar clinical performance of GIC and CR restorations concerning the marginal adaptation, marginal discoloration and anatomical form (Figs. 3-5), as well as the occurrence of failures (Figs. 2 and 6), indicates that both materials have similar adhesion capacity to the cavity walls, since this aspect is one of the main responsible for marginal sealing, which is directly related to the variables analyzed. Considering the different adhesion mechanisms of the materials studied, it was expected that different types of GIC might have influenced the results in relation to the aforementioned parameters, which was not confirmed after analysis of C-GIC and RM-GIC in subgroups. Therefore, it can be considered that the marginal sealing obtained by chemical adhesion to the tooth structure (C-GIC) is strong enough to assure similar longevity rates as those obtained by CR (micromechanical retention) or RM-GIC (chemical bond and micromechanical retention). It is noteworthy, however, that retention of the restorative material should not be only associated to adhesion, as other factors related to cavity design (i.e., cavity size, cavity type, and number of restored surfaces) are also known to play a significant role on the rate of failures [25].

Conversely, GIC restorations presented better performance than CR regarding the occurrence of secondary carious lesions, which seems to be related to the better physicochemical properties of GIC regarding biocompatibility, chemical bond to the tooth structure, similar thermal expansion coefficient compared to dentin, and mainly its ability to release and recharge fluoride $[26,27]$. Regarding the differences between C-GICs and RM-GICs, it has been reported that an increase in the amount of resin component and decrease in polyacid and glass filler content of the restorative material may lead to a decrease in fluoride release [28]. Therefore, it could be assumed that C-GICs would promote a higher protective effect considering secondary caries lesions compared with RM-GICs, thus the two types of GIC were individually analyzed in 2 MAs. In disagreement with this assumption, while restorations performed with RM-GIC presented better clinical performance than $\mathrm{CR}$ as to the occurrence of secondary caries (Fig. 7), no significant differences were observed between C-GIC and CR for this parameter (Fig. 8). Even though the reasons for these findings are not evident, it can be assumed that the reduced number of studies

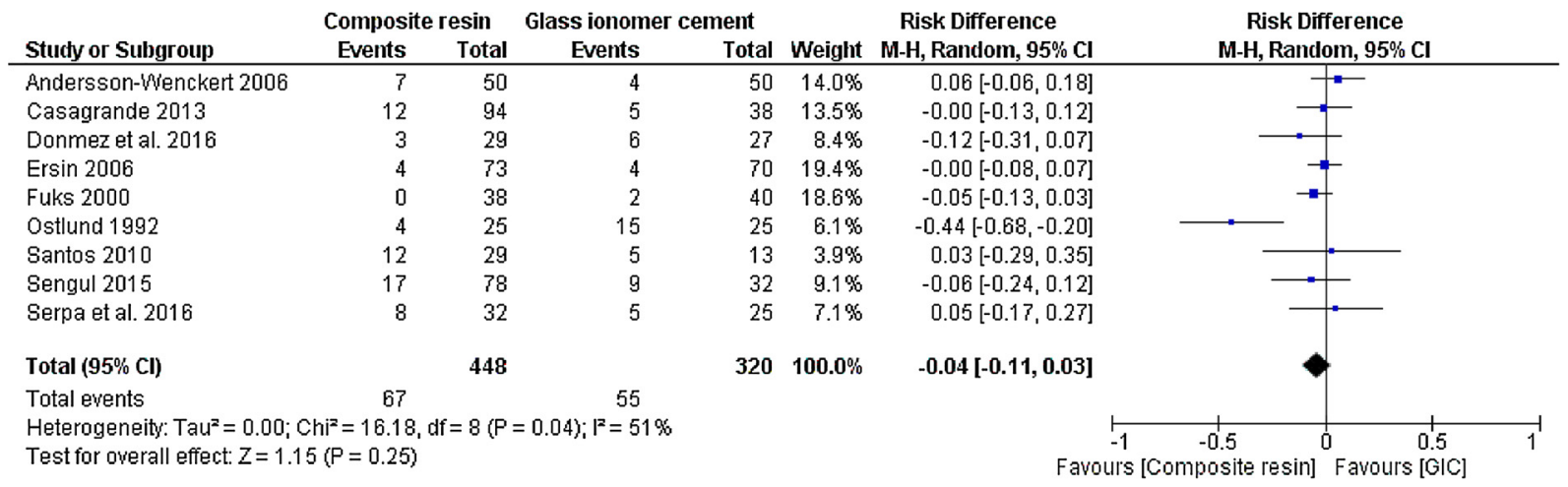

Fig. 2. Forest plot of failures of Class II restorations in primary teeth, performed with glass ionomer cement or composite resin. 


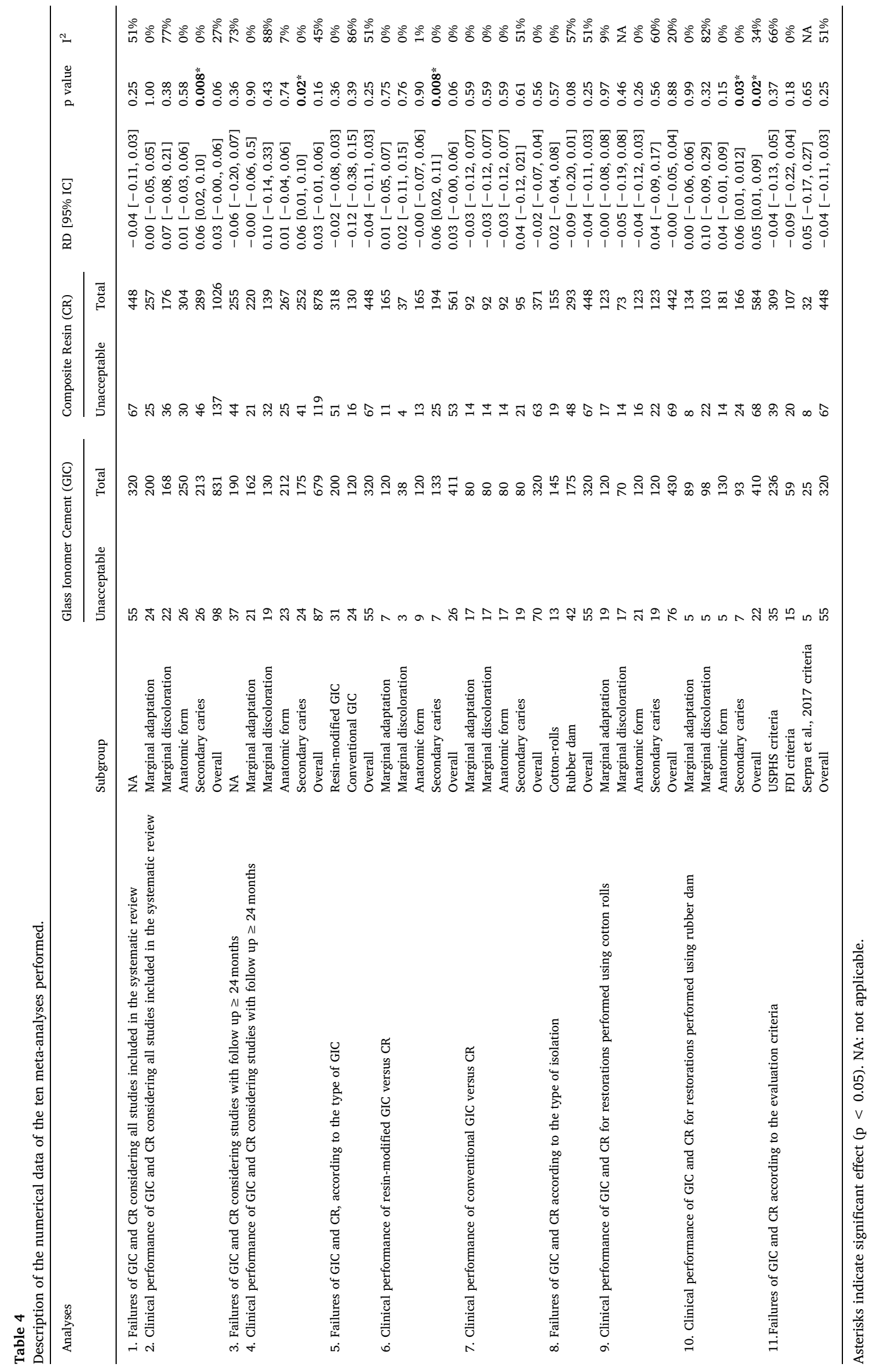




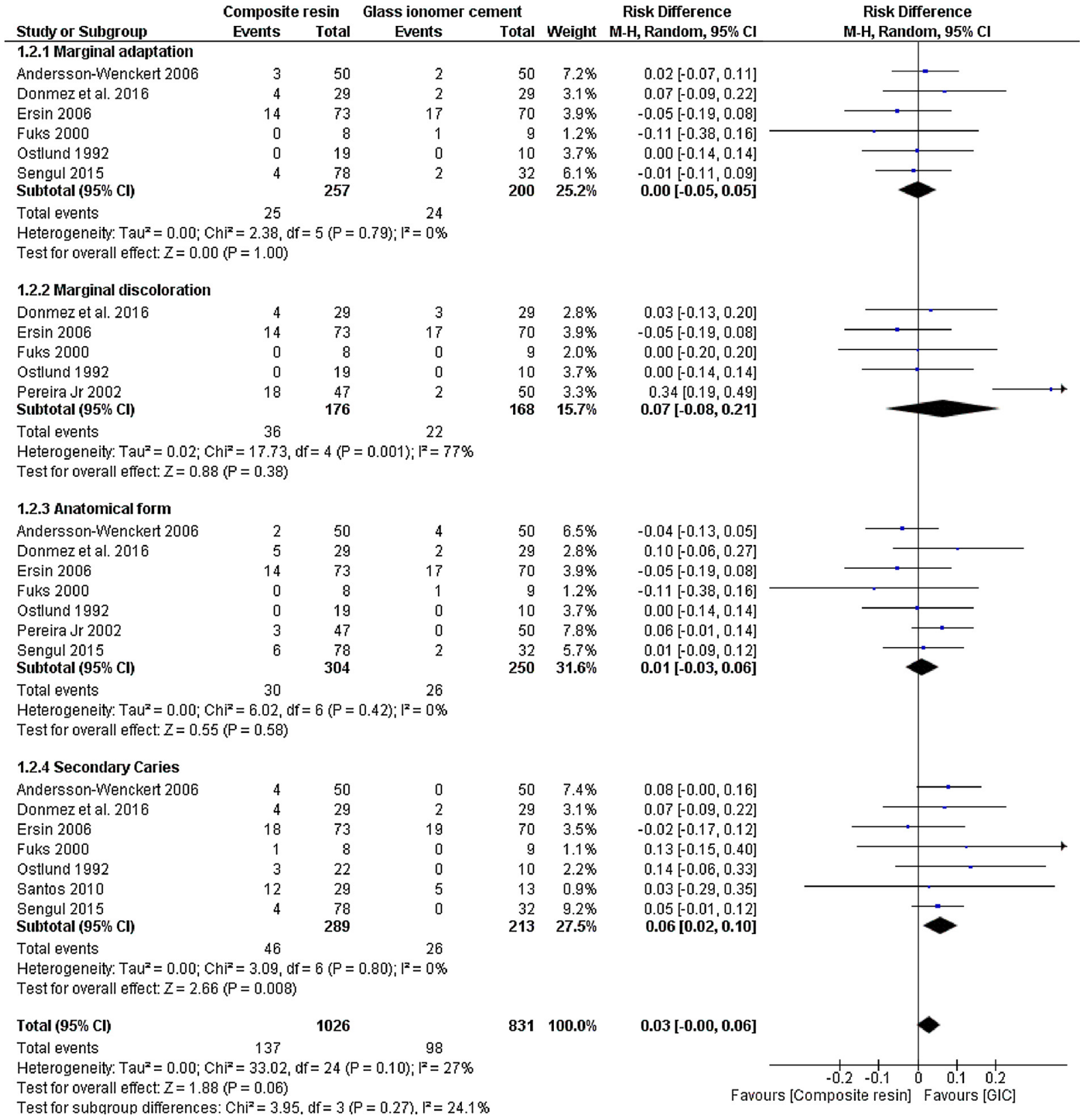

Fig. 3. Forest plot of clinical performance of Class II restorations in primary teeth, performed with glass ionomer cement or composite resin.

comparing C-GIC and CR (only 2 studies), the longer curing time and greater possibility of syneresis and imbibition of C-GIC (as compared to RM-GIC) [29], and the discrepant results among the studies included may have contributed for the trend observed. Two other important aspects should also be emphasized. Firstly, it has been reported that the ability of fluoride release and recharge is significantly reduced after material maturation [30]. Second - and most importantly - there is no consistent evidence from clinical trials assessing the caries-preventive effect of GIC restorations as a function of the amount of fluoride released from the materials. Thus, additional controlled clinical studies would be necessary to allow more consistent conclusions, especially for primary teeth.

Although the two types of GIC were not directly compared in this review, the superiority of RM-GIC compared to C-GIC had already been demonstrated in a previous meta-analysis [31], which reinforces the present data. However, it should be highlighted that the similarity between C-GIC and CR in the present study, in a meta-analysis conducted with results from three studies identified as eligible, is a positive finding, especially considering that the longevity of occluso-proximal restorations with C-GIC has been reported to be shorter compared to CR in isolated studies [21]. Conversely, the superiority of RM-GIC as to the occurrence of secondary caries suggests that this material satisfactorily combines the adhesive properties of CRs and GICs, the cariostatic effects of $\mathrm{F}$ release to the restoration margins, besides the advantages of light-curing, which significantly reduces the early sensitivity of GIC to moisture and dehydration [32].

Another aspect considered in the present review was the follow-up period of restorations. The studies included presented follow-up periods ranging from 6 to 48 months, which could contribute to the heterogeneity of studies. Considering that the exclusion of studies with followup period shorter than 24 months did not affect the heterogeneity of MAs regarding the rate of failures or the clinical performance of the materials analyzed, it may be concluded that the great variation in the follow-up periods in the present review had little or no influence on the outcomes.

Among the factors contributing to the greater longevity of restorations 


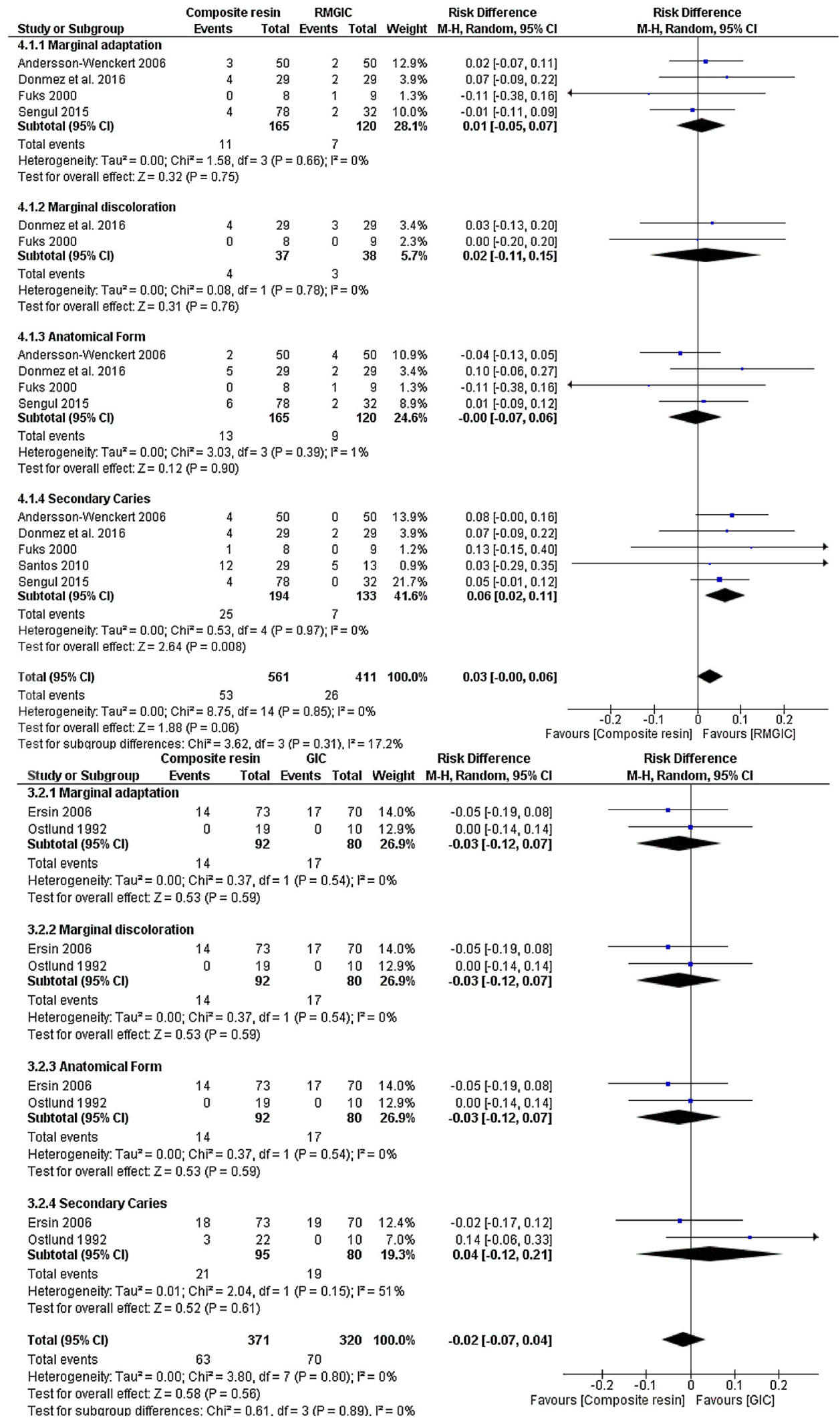

Fig. 4. Forest plot of clinical performance of Class II restorations in primary teeth, performed with glass ionomer cement or composite resin, including only studies using resin-modified (above) or conventional (below) glass ionomer cement.

in posterior teeth, effective moisture control during the procedure is considered paramount. This aspect is even more critical for occlusoproximal restorations, since they are susceptible to contamination both from saliva and from the gingival fluid. However, systematic literature reviews present conflicting information regarding the influence of the type of isolation on the longevity of restorations $[33,34]$, therefore the influence of the type of isolation was also assessed in the present study. The meta-analysis of studies using rubber dam isolation revealed similar 


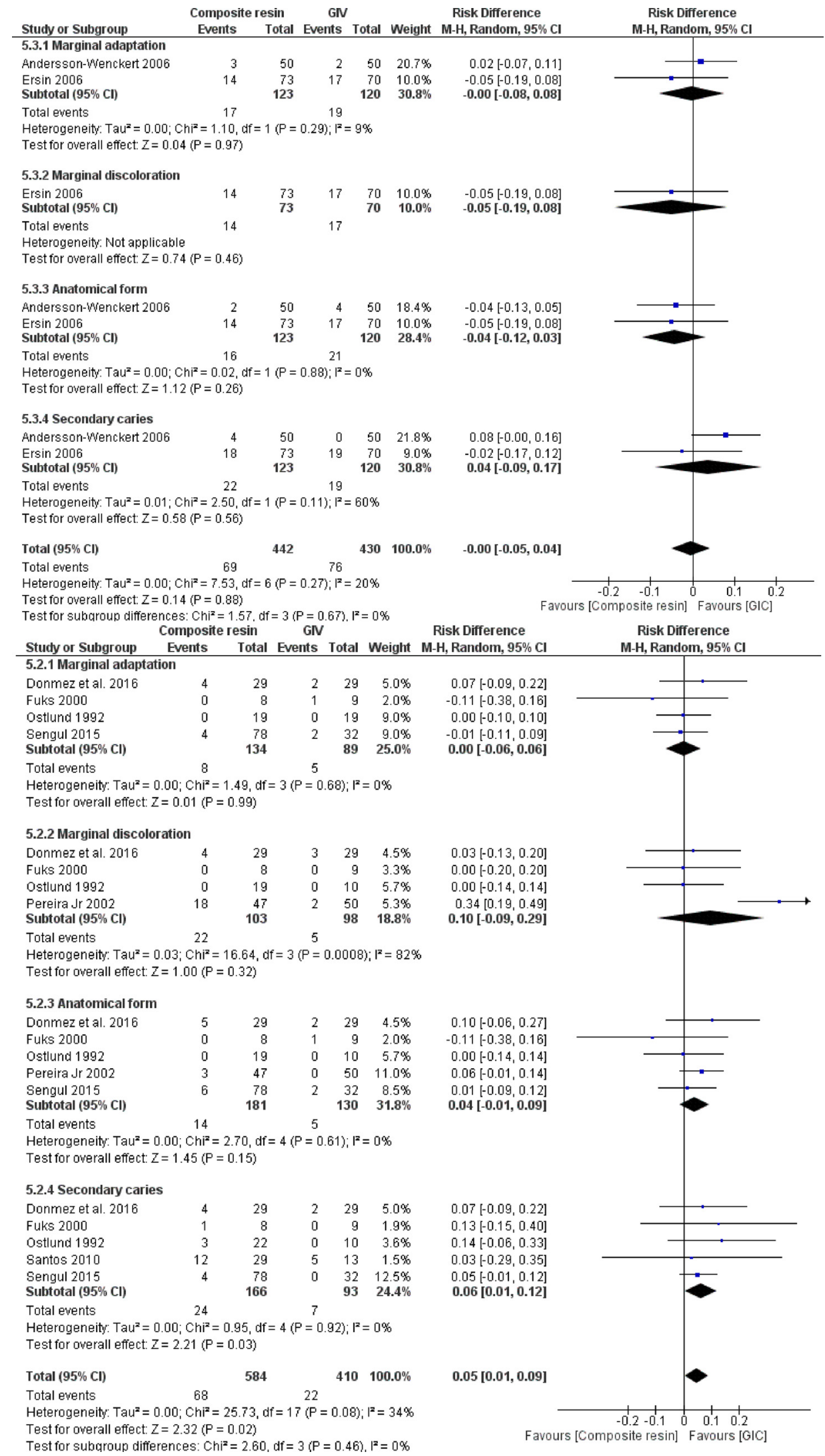

Fig. 5. Forest plot of clinical performance of Class II restorations in primary teeth, performed with glass ionomer cement or composite resin, including only studies using cotton roll (above) or rubber dam (below). 


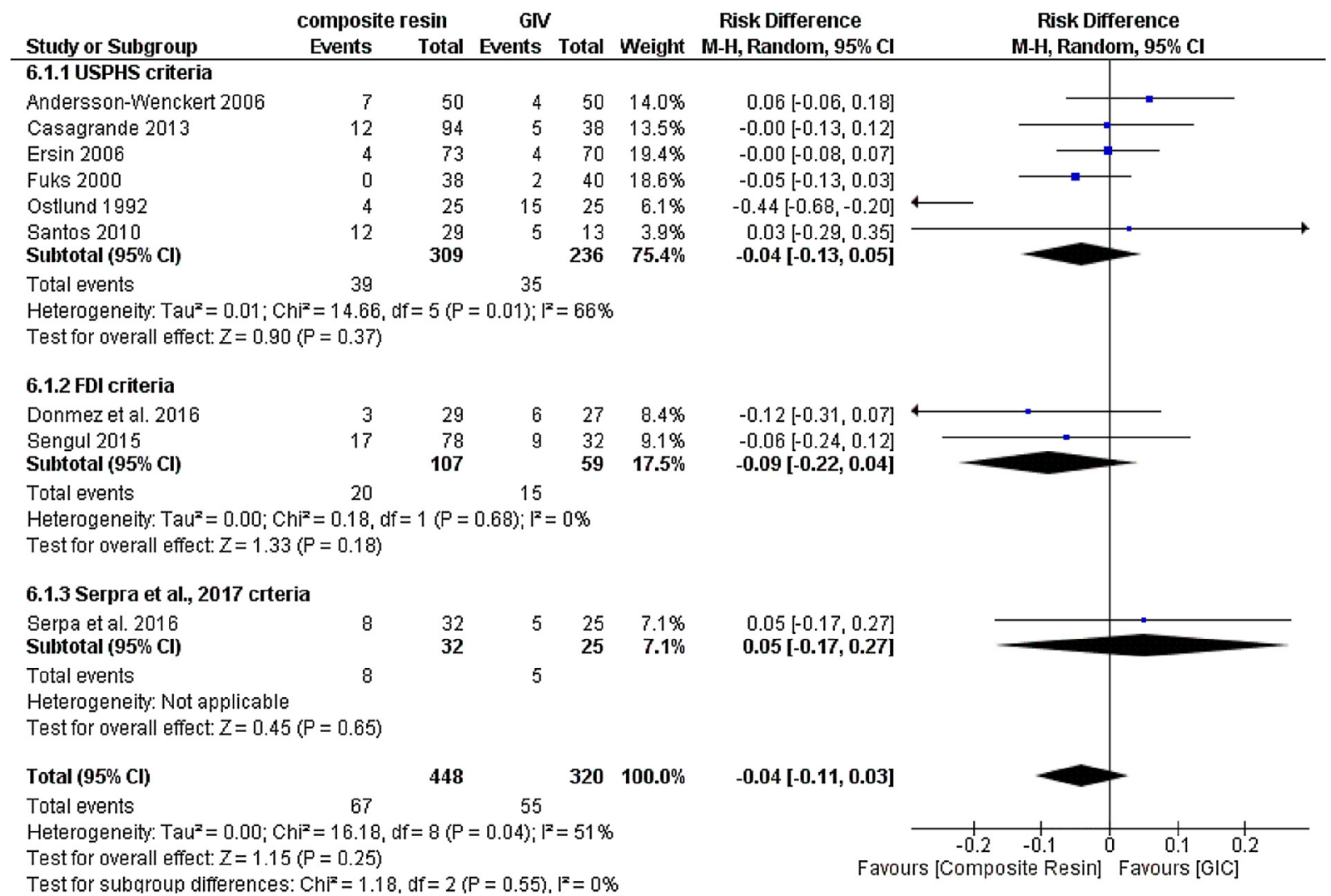

Fig. 6. Forest plot of failures of Class II restorations in primary teeth, evaluation with USPHS criteria, FDI criteria and Serpra et al., 2017 criteria.

findings as the meta-analysis of clinical performance considering all studies together (i.e., GIC only presented better results concerning the occurrence of secondary caries lesions). However, for studies using cotton roll isolation, no difference was found between materials for any of the parameters assessed. Since the reasons for such findings are not evident, and considering the inconsistencies concerning the influence of the type of isolation on the longevity of adhesive restorations [33,34], it is clear that further studies with adequate protocols are required to address this important clinical issue.

A meta-analysis presents the advantage to gather information from several primary studies, which assigns greater statistical power compared to less accurate results from a single primary study [35]. However, this method has some flaws, since it is not possible to control sources of bias from individual studies. In the present study, besides the aspects previously discussed, some factors may also have contributed to the heterogeneity among the studies, especially the individual characteristics of participants and populations of the studies included, as well as the clinical skills and calibration of operators and examiners. Additionally, inherent differences to each type of restorative material may have had some influence on the results. Thus, considering that the included studies were published between 1992 and 2017, it is likely that the studies published more recently used materials with better physical, mechanical and biological properties, given that restorative materials are in constant evolution.

Finally, it should be observed that the similarity between GIC and CR in the analysis of longevity ("overall effect"), regardless of the type of GIC, is a clinically relevant finding, since Class II restorations performed with GIC are often associated with shorter longevity than CR $[12,13]$ due to the aforementioned reasons (e.g., greater interface area between the tooth and the restorative material, and loss of the marginal ridge) [14]. The present findings allow professionals greater freedom of choice among the restorative materials most widely available for direct restorations, considering important clinical parameters related to marginal adaptation and wear of the restorative material. Thus, professionals can consider other operational aspects, such as child behavior, curing time of the material, and possibility of using rubber dam isolation before selecting the restorative material.

\section{Conclusions}

The results allowed to conclude that the materials analyzed (GIC and CR) presented similar clinical performance to each other concerning the percentage of failures, marginal adaptation, marginal discoloration and anatomical form in Class II restorations in primary teeth, regardless of the type of GIC or isolation. However, regarding the occurrence of secondary carious lesions, GIC presented superior clinical performance, and this effect was more evident for the resin-modified GIC used with rubber dam isolation. Therefore, the possibility of light curing, combined with its cariostatic properties, indicate that resinmodified GIC is a suitable material for Class II restorations in primary teeth. The high number of subgroups and the low number of studies included, however, are important limitations of the present review, emphasizing the need for well-controlled clinical trials addressing relevant variables related to the longevity of restorations in the primary dentition.

\section{Acknowledgements}

For their help in this systematic review, the authors would like to thank to Anna Blinder Fuks, Melek Turgut, Karin Sunnegårdh Grönberg, Edmer Silvestre Pererira Jr, Luciano Casagrande and Marcia P. Alves dos Santos, who kindly provided information not published in their studies. 


\section{References}

[1] M.A. Peres, A. Sheiham, P. Liu, F.F. Demarco, A.E. Silva, M.C. Assuncao, et al, Sugar consumption and changes in dental caries from childhood to adolescence, J. Dent. Res. 95 (4) (2016) 388-394.

[2] I. Mejare, H. Stenlund, C. Zelezny-Holmlund, Caries incidence and lesion progression from adolescence to young adulthood: a prospective 15-year cohort study in Sweden, Caries Res. 38 (2) (2004) 130-141.

[3] T.F. Novaes, R. Matos, M.M. Braga, J.C. Imparato, D.P. Raggio, F.M. Mendes, Performance of a pen-type laser fluorescence device and conventional methods in detecting approximal caries lesions in primary teeth-in vivo study, Caries Res. 43 (1) (2009) 36-42.

[4] K.R. Ekstrand, L.E. Luna, L. Promisiero, A. Cortes, S. Cuevas, J.F. Reyes, et al., The reliability and accuracy of two methods for proximal caries detection and depth on directly visible proximal surfaces: an in vitro study, Caries Res. 45 (2) (2011) 93-99.

[5] J.Y. Wong, Low-quality evidence suggests that amalgam has increased longevity compared with resin-based composite in posterior restorations, J. Am. Dent. Assoc. 147 (11) (2016) 905-906.

[6] M.G. Rasines Alcaraz, A. Veitz-Keenan, P. Sahrmann, P.R. Schmidlin, D. Davis, Z. Iheozor-Ejiofor, Direct composite resin fillings versus amalgam fillings for permanent or adult posterior teeth, Cochrane Database Syst. Rev. 31 (3) (2014) CD005620.

[7] A.B. Fuks, The use of amalgam in pediatric dentistry: new insights and reappraising the tradition, Pediatr. Dent. 37 (2) (2015) 25-32.

[8] J.H. Berg, T.P. Croll, Glass ionomer restorative cement systems: an update, Pediatr. Dent. 37 (2) (2015) 16-24.

[9] M. Almuhaiza, Glass-ionomer cements in restorative dentistry: a critical appraisal, J. Contemp. Dent. Pract. 17 (4) (2016) 331-336.

[10] A.P. Santos, I.K.D. Moreira, A.C. Scarpelli, I.A. Pordeus, S.M. Paiva, C.C. Martins, Survival of adhesive restorations for primary molars: a systematic review and metaanalysis of clinical trials, Pediatr. Dent. 38 (5) (2016) 370-378.

[11] D.P. Raggio, T.K. Tedesco, A.F. Calvo, M.M. Braga, Do glass ionomer cements prevent caries lesions in margins of restorations in primary teeth?: A systematic review and meta-analysis, J. Am. Dent. Assoc. 147 (3) (2016) 177-185.

[12] M.P.A. Santos, M. Passos, R.R. Luiz, L.C. Maia, A randomized trial of resin-based restorations in class $i$ and class ii beveled preparations in primary molars: 24-month results, J. Am. Dent. Assoc. 140 (2) (2009) 156-166.

[13] M.P.A. Santos, R.R. Luiz, L.C. Maia, Randomised trial of resin-based restorations in Class I and Class II beveled preparations in primary molars: 48-month results, J. Dent. 38 (6) (2010) 451-459.

[14] R.J. Simonsen, Conservation of tooth structure in restorative dentistry, Quintessence Int. 4 (1) (1985) 15-24.

[15] D. Moher, A. Liberati, J. Tetzlaff, D.G.P. Altman, Preferred reporting items for systematic reviews and meta-analyses: the PRISMA statement, Ann. Intern. Med. 151 (4) (2009) 264-269.

[16] I. Andersson-Wenckert, K. Sunnegardh-Gronberg, Flowable resin composite as a class II restorative in primary molars: a two-year clinical evaluation, Acta Odontol. Scand. 64 (6) (2006) 334-340.

[17] L. Casagrande, D.M. Dalpian, T.M. Ardenghi, F.B. Zanatta, C.E. Balbinot, F. GarciaGodoy, et al., Randomized clinical trial of adhesive restorations in primary molars. 18-month results, Am. J. Dent. 26 (6) (2013) 351-355.
[18] S. Bektas Donmez, S. Uysal, A. Dolgun, M.D. Turgut, Clinical performance of aesthetic restorative materials in primary teeth according to the FDI criteria, Eur. J. Paediatr. Dent. 17 (3) (2016) 202-212.

[19] N.K. Ersin, U. Candan, A. Aykut, O. Oncag, C. Eronat, T. Kose, A clinical evaluation of resin-based. composite and glass ionomer cement restorations placed in primary teeth using the ART approach: results at 24 months, J. Am. Dent. Assoc. 137 (11) (2006) 1529-1536.

[20] A.B. Fuks, F.B. Araujo, L.B. Osorio, P.E. Hadani, A.S. Pinto, Clinical and radiographic assessment of Class II esthetic restorations in primary molars, Pediatr. Dent. 22 (6) (2000) 479-485.

[21] J. Ostlund, K. Moller, G. Koch, Amalgam, composite resin and glass ionomer cement in class-ii restorations in primary molars: a three year clinical evaluation, Swed. Dent. J. 16 (3) (1992) 81-86.

[22] E.S. Pereira Jr., Two years the clinical, radiographic and marginal microleakage performance of a resin-modified glass ionomer cement and the "condensable" composite resin in the restoration of class II cavities in the primary molars [tese], Universidade de São Paulo, Bauru, 2002.

[23] F. Sengul, T. Gurbuz, Clinical evaluation of restorative materials in primary teeth class II lesions, J. Clin. Pediatr. Dent. 39 (4) (2015) 315-321.

[24] E.B.M. Serpa, M.A. Clementino, A.F. Granville-Garcia, A. Rosenblatt, The effect of atraumatic restorative treatment on adhesive restorations for dental caries in deciduous molars, J. Indian Soc. Pedod. Prev. Dent. 35 (2) (2017) 167-173.

[25] F.F. Demarco, M.B. Corrêa, M.S. Cenci, R.R. Moraes, N.J. Opdam, Longevity of posterior composite restorations: not only a matter of materials, Dent. Mater. 28 (1) (2012) 87-101.

[26] M.J. Tyas, Milestones in adhesion: glass-ionomer cements, J. Adhes. Dent. 5 (4) (2003) 259-266.

[27] M.J. Tyas, M.F. Burrow, Adhesive restorative materials: a review, Aust. Dent. J. 49 (3) (2004) 112-121.

[28] A.G. Nigam, J. Jaiswal, R. Murthy, R. Pandey, Estimation of fluoride release from various dental materials in different media-an in vitro study, Int. J. Clin. Pediatr. Dent. 2 (1) (2009) 1-8.

[29] C.L. Davidson, I.A. Mjör, Advances in Glass Ionomer Cements, Quintessence, Carol Stream, 1999.

[30] S.K. Sidhu, J.W. Nicholson, A review of glass-ionomer cements for clinical dentistry, J Funct Biomater. 7 (3) (2016) pii: E16.

[31] B.L. Chadwick, D.J. Evans, Restoration of class II cavities in primary molar teeth with conventional and resin modified glass ionomer cements: a systematic review of the literature, Eur. Arch. Paediatr. Dent. 8 (1) (2007) 14-21.

[32] E. Bresciani, T.J.E. Barata, T.C. Fagundes, A. Adachi, M.M. Terrin, M.F.L. Navarro, Compressive and diametral tensile strength of glass ionomer cements, J. Appl. Oral Sci. 12 (4) (2004) 344-348.

[33] M.R. Cajazeira, T.M. De Sabóia, L.C. Maia, Influence of the operatory field isolation technique on tooth-colored direct dental restorations, Am. J. Dent. 27 (3) (2014) 155-159.

[34] Y. Wang, C. Li, H. Yuan, M.C. Wong, J. Zou, Z. Shi, et al., Rubber dam isolation for restorative treatment in dental patients, Cochrane Database Syst. Rev. 9 (2016) CD009858.

[35] J.P.T. Higgins, D.G.D. Altman, P.C. Gotzsche, P. Jüni, D. Moher, A.D. Oxman, et al., The Cochrane Collaborationüs tool for assessing risk of bias in randomised trials, Br. Med J. 343 (2011) d5928. 